\title{
Combination of TP53 and AGR3 to distinguish ovarian high-grade serous carcinoma from low-grade serous carcinoma
}

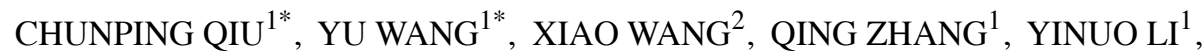 \\ YING XU $^{1}$, CHENGJUAN JIN ${ }^{1}$, HUALEI BU ${ }^{1}$, WENXIN ZHENG ${ }^{3-5}$, \\ XINGSHENG YANG ${ }^{1}$, NAN LU ${ }^{6}$ and BEIHUA KONG ${ }^{1}$
}

\author{
${ }^{1}$ Department of Obstetrics and Gynecology, Qilu Hospital of Shandong University; ${ }^{2}$ Department of Pathology, \\ School of Medicine, Shandong University, Jinan, Shandong 250012, P.R. China; Departments of ${ }^{3}$ Pathology \\ and ${ }^{4}$ Obstetrics and Gynecology, ${ }^{5}$ Harold C. Simmons Comprehensive Cancer Center, \\ University of Texas Southwestern Medical Center, Dallas, TX 75390-9072, USA; \\ ${ }^{6}$ Department of Diagnostics, School of Medicine, Shandong University, \\ Jinan, Shandong 250012, P.R. China
}

Received December 11, 2017; Accepted March 19, 2018

DOI: $10.3892 /$ ijo.2018.4360

\begin{abstract}
Ovarian high-grade serous carcinoma (HGSC) and low-grade serous carcinoma (LGSC) are distinct gynecologic neoplasms with diverse pathogenesis and characteristic features. They respond differently to same modalities of treatment protocol and have dissimilar prognosis. Thus, it is essential to obtain accurate differential diagnosis of HGSC and LGSC prior to clinical treatment. In the present study, mRNA expression profiles were generated from 5 HGSC and 6 LGSC specimen using HTSeq, and 699 differentially expressed genes ( $>2$-fold difference) were identified using the DESeq R package. Dendrograms produced by unsupervised hierarchical clustering completely distinguished HGSC from
\end{abstract}

Correspondence to: Dr Beihua Kong, Department of Obstetrics and Gynecology, Qilu Hospital of Shandong University, 107 Wenhua Xi Road, Jinan, Shandong 250012, P.R. China

E-mail: kongbeihua@sdu.edu.cn

Professor Nan Lu, Department of Diagnostics, School of Medicine, Shandong University, 44 Wenhua Xi Road, Jinan, Shandong 250012, P.R. China

E-mail: lunan0501@163.com

${ }^{*}$ Contributed equally

Abbreviations: HGSC, high-grade serous carcinoma; LGSC, low-grade serous carcinoma; AGR3, anterior gradient homolog 3; ROC, receiver operating characteristic; EOC, epithelial ovarian cancer

Key words: high-grade serous carcinoma, low-grade serous carcinoma, differential diagnosis, tumor protein 53, anterior gradient homolog 3
LGSC. Among differentially expressed genes between HGSC and LGSC, anterior gradient homolog 3 (AGR3) was highly upregulated in LGSC compared to HGSC, which was validated by reverse transcription-quantitative polymerase chain reaction and western blotting. Then, anti-tumor protein 53 (TP53) and anti-AGR3 immunohistochemistry were performed on 145 HGSC and 30 LGSC samples. Consistent with previous studies, abnormal expression of TP53 ( 0 or $\geq 75 \%$ positive expression) was observed in $87.6 \%$ of HGSC and $13.3 \%$ of LGSC samples. Positive staining of AGR3 had a sensitivity of $80.0 \%$ and specificity of $89.7 \%$ for LGSC. TP53 and AGR3 were both efficient in distinguishing HGSC from LGSC $(\mathrm{P}<0.001)$. Receiver operating characteristic analysis revealed a similar area under the curve for AGR3 (0.848) and TP53 (0.871). Through combination of the two markers (TP53 wild-type pattern and AGR3-positive expression), the accuracy of differential diagnosis was up to $93.1 \%$. These findings provide compelling evidence that differential diagnosis of HGSC and LGSC can be improved by combined application of these two markers on the basis of conventional histopathological diagnosis.

\section{Introduction}

Epithelial ovarian cancer (EOC) is the seventh most common cause of tumor-associated mortality in women and the most lethal gynecologic malignancy. The American Cancer Society estimated a total of 22,440 new cases and 14,080 mortalities from the disease in 2017 (1). The high fatality rate is predominantly attributed to the late detection and chemoresistance of EOC. It is a heterogeneous condition composed of different types of tumors with widely varied clinicopathological features and behavior. EOC, according to histological criteria, is divided into five major subtypes: High-grade serous, clear cell, endometrioid, mucinous and low-grade serous types. These five subtypes 
are best considered to be distinct entities, of which serous type, accounting for $70 \%$ of EOC, is the most common $(2,3)$.

Histological grade has been demonstrated to be an important prognostic factor for serous ovarian carcinoma in previous studies (4-7). However, there are several traditional systems used for grading these tumors with different categories and number of strata (International Federation of Gynecology and Obstetrics, World Health Organization and Gynecologic Oncology Group criteria) (8). In 2002 and subsequently in 2004, a two-tier system for grading serous ovarian carcinoma was proposed, according to which tumors are subdivided into high-grade and low-grade $(4,9)$. Although a large number of studies report that high-grade serous carcinoma (HGSC) and low-grade serous carcinoma (LGSC) share the same origin from fallopian tubal epithelia, they are completely distinct types of gynecologic tumor, rather than different grades of the same neoplasm, and there are drastic clinicopathological and molecular differences between the two (10-13). HGSC is usually susceptible to platinum-based chemotherapy; however 5- and 10-year survival rates for advanced staged carcinoma are $\sim 25$ and $0 \%$, respectively. LGSC progresses slowly with a 5 -year survival rate of $85 \%$ and a 10 -year survival rate of $50 \%$. It is usually resistant to standard chemotherapy. Thus, optimal cytoreductive surgery is more critical for the treatment of LGSC (14). Accurate differential diagnosis may help to reduce unnecessary morbidity and optimize therapeutic effectiveness.

Primary cytoreductive surgery has been considered the standard therapeutic strategy for serous ovarian cancer in many centers, however neoadjuvant chemotherapy followed by interval debulking surgery is achieving more and more attention recently. A clinical trial conducted by Vergote et al (15) among patients with stage IIIC and IV ovarian cancers demonstrated that there was no difference in survival rates between patients undergoing primary cytoreductive surgery and those receiving neoadjuvant chemotherapy followed by interval debulking surgery. With respect to morbidity and mortality risk associated with the extent of surgery, neoadjuvant chemotherapy is preferable. Thus, neoadjuvant chemotherapy is quickly becoming the superior approach for advanced stage ovarian carcinoma. Unlike LGSC, HGSC is comparatively more responsive to chemotherapy. In addition, clinical trials specifically targeting the crucial molecular alterations of LGSC, such as CI-1040 targeting the mitogen-activated protein kinase pathway, have been launched and are on-going (16). Thus, a precise histopathology-based differential diagnosis of HGSC and LGSC is crucial for decision making by gynecological oncologists prior to any treatment.

The two-tier system for grading serous ovarian carcinoma is based primarily on nuclear atypia, with the mitotic rate used as a secondary criteria. Malpica et al (17) demonstrated that the system is easy to follow and is reproducible. In most epithelial ovarian tumors, the nuclear atypia of LGSC and HGSC corresponds to grade 1 and grade 3, respectively, in a three-grade system. However, nuclear features of certain tumors ( $4 \%$ of serous carcinomas) are intermediary between LGSC and HGSC (18). Thus, distinguishing these tumors of intermediate grade remains a challenge. Furthermore, subjective judgments on the criteria of nuclear atypia and mitotic rate from individual pathologists may also pose another problem for differential diagnosis of HGSC and LGSC. Thus, it is necessary to explore and establish objective criteria to assist differential diagnosis. In recent years, a number of researchers have begun to concentrate on distinguishing HGSC from LGSC through various methods, such as immunohistochemical markers (19). Although these methods do improve the accuracy of diagnosis to a certain extent, there have been no satisfactory results obtained as of yet. Thus, the purpose of the current study was to investigate useful markers in order to raise the efficacy in distinguishing HGSC from LGSC.

\section{Materials and methods}

Tissue samples for RNA-seq analysis. Flash-frozen primary serous ovarian tumor samples $(n=12)$ were collected from Qilu Hospital of Shandong University (Jinan, China) from patients that did not undergo preoperative chemotherapy: 6 ovarian high-grade serous cancers and 6 ovarian low-grade serous cancers. In the present study, patients with primary ovarian serous carcinoma were selected for investigation. Patients with other malignancies or those that had undergone preoperative chemotherapy were excluded. The ages of selected patients with 43-63 years. The samples were collected with the approval of the Ethics Committee at Qilu Hospital of Shandong University between January 2014 and December 2015, and with the signed informed consent from all patients. All tumors were diagnosed according to the two-tier system of MD Anderson Cancer Center criteria (4). Frozen sections (5 $\mu \mathrm{m})$ were cut, fixed onto slides, and immediately stored at $-20^{\circ} \mathrm{C}$. The remaining samples were immediately stored at liquid nitrogen for subsequent arrays. The slides were then stained with hematoxylin $(0.2 \%, 5 \mathrm{~min})$ and eosin $(1 \%, 10 \mathrm{~min})$ at room temperature for reviewing and ensuring accurate diagnosis by two gynecological pathologists. Tumor samples containing $>70 \%$ of invasive cancer were used for sequencing analysis.

RNA extraction and RNA-seq analysis. Total RNA was isolated from cancer samples using TRIzol reagent (Thermo Fisher Scientific, Inc., Waltham, MA, USA) according to the manufacturer's protocol. Prior to mRNA sequencing, the quality of all RNA samples were detected by agarose gel (1\%) electrophoresis and Agilent 2100 bioanalyzer (Agilent Technologies, Inc., Santa Clara, CA, USA). Following mRNA sequencing using the Illumina HiSeq X Ten platform, TopHat2 (ccb.jhu.edu/software/tophat) was used to align paired-end clean reads to the reference genome (20). One HGSC sample was excluded because of lower proportion of total reads mapped (<80\%). Then, HTSeq v0.9.1 (pypi.python.org/pypi/ HTSeq) was applied to count the read numbers mapped of each gene. Subsequently, reads per kilobase of exon model per million mapped reads (RPKM) of each gene was calculated based on the length of the gene and reads count mapped to this gene. RPKM takes sequencing depth and gene length into consideration and is currently the most common method for estimating gene expression levels (21).

Differential expression analysis and clustering. Differential expression analysis between HGSC and LGSC was performed using the DESeq R package (version 3.4.2; r-project.org/). DESeq provides statistical routines for testing differential expression by the use of negative binonial distribution. $\mathrm{P}<0.05$ in DESeq 
was considered to indicate a statistically significant difference. To identify the correlation of different samples, differentially expressed genes were clustered between HGSC and LGSC using unsupervised hierarchical clustering method with the function of heatmap. 2 in $\mathrm{R}$ (version 3.4.2). Manhattan distance metric with average linkage was used for clustering. To allow for log adjustment, genes with 0 RPKM were assigned a value of 0.01 . We also performed a Gene Ontology analysis (geneontology.org) on all the differential genes between HGSC and LGSC.

Reverse transcription-quantitative polymerase chain reaction $(R T-q P C R)$ analysis. Ovarian HGSC $(\mathrm{n}=36)$ and LGSC $(\mathrm{n}=15)$ were used for validation of differentially expressed genes between them in mRNA sequencing. They were obtained from Qilu Hospital of Shandong University between January 2011 and June 2016. The ages of selected patients are range from 41-76 years. The inclusion and exclusion criteria of selected patients were the same as described above. Tumor samples containing $>70 \%$ of invasive cancer were selected for research. Based on the above results and the literature, a number of molecules were selected for further validation and investigation [lysosomal associated membrane protein 3 (LAMP3), EGF like domain multiple 6 (EGFL6), cyclin-dependent kinase inhibitor 1A (CDKN1A), anterior gradient 3, protein disulphide isomerase family member (AGR3), glutathione S-transferase $\mu 1$ (GSTM1), tectonic family member 1 (TCTN1), spermatogenesis associated 18 (SPATA18)]. Of them, LAMP, EGFL6, CDKN1A and AGR3 exhibited significant differences between HGSC and LGSC, and were selected in the current study to demonstrate the results. Oligonucleotide primers (Sangon Biotech Co., Ltd., Shanghai, China) designed for each gene were as follows: LAMP3 forward, 5'-GCGTCCCT GGCCGTAATTT-3' and reverse, 5'-TGCTTGCTTAGCTGG TTGCT-3'; EGFL6 forward, 5'-GTCTGTGAAGCTACAT GCGAA-3' and reverse, 5'-CATGGCCGGGGTTTCATTC-3'; CDKN1A forward, 5'-CGATGGAACTTCGACTTTGTCA-3' and reverse, 5'-GCACAAGGGTACAAGACAGTG-3'; AGR3 forward, 5'-ATCACCTGATGGGCAATATGTG-3' and reverse, 5'-GAGTATCTTCCAGCTATGTCAGC-3'; $\beta$-actin forward, 5'-TCATGAAGTGTGACGTGGACATC-3' and reverse, 5'-CAGGAGGAGCAATGATCTTGATCT-3'. $\beta$-actin was used as the endogenous control.

Each RNA sample $(<1 \mu \mathrm{g})$ was reverse transcribed using the Reverse Transcription system (Takara Bio, Inc., Otsu, Japan) in $20 \mu \mathrm{l}$ reaction system. Following $37^{\circ} \mathrm{C}$ incubation for $15 \mathrm{~min}$, the reaction system was incubated in $85^{\circ} \mathrm{C}$ for $5 \mathrm{sec}$ and then stored at $-20^{\circ} \mathrm{C}$. RT-qPCR was performed using SYBR-Green qPCR master mix (Takara Bio, Inc.), with the ROX as internal reference dye. PCR was conducted using the ABI PRISM 7900HT System (Applied Biosystems; Thermo Fisher Scientific, Inc.). The qPCR cycling conditions were as follows: Predegeneration at $95^{\circ} \mathrm{C}$ for $30 \mathrm{sec}$, then denaturation at $95^{\circ} \mathrm{C}$ for $5 \mathrm{sec}$ and annealing at $60^{\circ} \mathrm{C}$ for $34 \mathrm{sec}$, the last two steps were repeated for a total of 40 cycles. All experiments were performed in triplicate for target and reference genes. Statistical analysis was conducted using t-test. $\mathrm{P}<0.05$ was considered to indicate a statistically significant difference.

Western blot analysis. HGSC $(\mathrm{n}=8)$ and seven LGSC $(\mathrm{n}=7)$ samples were included for this analysis. They were obtained
Table I. Main clinicopathological characteristics of patients enrolled in the immunohistochemistry study.

\begin{tabular}{lll}
\hline Characteristic & HGSC $(\mathrm{n}=145)$ & LGSC $(\mathrm{n}=30)$ \\
\hline $\begin{array}{l}\text { Age at diagnosis } \\
\text { Mean } \pm \text { SD }\end{array}$ & $55.43 \pm 9.74$ & $50.20 \pm 11.48$ \\
Median (range) & $56(35-78)$ & $49(24-77)$ \\
FIGO stage (n) & & \\
I+II & 30 & 13 \\
III+IV & 115 & 17 \\
CA125 (n) & & \\
$<200 \mathrm{U} / \mathrm{ml}$ & 20 & 14 \\
$\geq 200 \mathrm{U} / \mathrm{ml}$ & 125 & 16 \\
\hline
\end{tabular}

SD, standard deviation; FIGO, International Federation of Gynecology and Obstetrics; CA125, cancer antigen 125.

from Qilu Hospital of Shandong University from January 2010 to December 2014. The ages of selected patients range from 43-76 years. The inclusion and exclusion criteria of selected patients were as described above. All samples were cut into pieces before they were lysed on ice with radioimmunoprecipitation assay buffer (Biocolor Ltd., Carrickfergus, UK) and protease inhibitors. The protein concentration was determined using a bicinchoninic acid assay kit (Beyotime Institute of Biotechnology, Haimen, China). Protein samples (30 $\mu \mathrm{g})$ were separated using 12\% (separating gel) and 6\% (stacking gel) SDS-polyacrylamide gel and then electrotransferred onto polyvinylidene difluoride membrane (EMD Millipore, Billerica, MA, USA). Following blocking with 5\% non-fat milk at room temperature for $1 \mathrm{~h}$, the membranes were incubated overnight at $4^{\circ} \mathrm{C}$ with the mouse anti-human monoclonal antibody against AGR3 (1:1,000; cat. no. ab82400; Abcam, Cambridge, UK), mouse anti-human monoclonal antibody against tumor protein 53 (TP53; 1:1,000; cat. no. M7001; Dako; Agilent Technologies, Inc.) and mouse anti-human monoclonal antibody against $\beta$-actin (1:2,000; cat. no. A2228; Sigma-Aldrich; Merck KGaA, Darmstadt, Germany). Subsequently, the membranes were incubated with specific horseradish peroxidase-labeled secondary antibodies to mouse $\mathrm{IgG}$ (1:5,000; cat. no. 074-1806; Kirkegaard \& Perry Laboratories Inc., Gaithersburg, MD, USA) at room temperature for $1 \mathrm{~h}$. Signal was detected with enhanced chemiluminescence using Western Lighting Plus ECL (PerkinElmer, Inc., Waltham, MA, USA) by ImageQuant LAS 4000 (GE Healthcare Life Sciences, Little Chalfont, UK).

Immunohistochemistry (IHC) analysis. Protein expression of AGR3 and TP53 was detected in samples of HGSC $(n=145)$ and LGSC $(n=30)$. Cases were reviewed by two gynecological pathologists, and a diagnosis of high and low-grade serous ovarian carcinomas was established according to the two-tier system of MD Anderson Cancer Center criteria. The clinicopathologic characteristics of all samples are shown in Table I. Formalin-fixed (10\% formalin fixation for $24-48 \mathrm{~h}$ at room temperature) and paraffin-embedded tissues were sectioned at 
$4 \mu \mathrm{m}$ and incubated at $70^{\circ} \mathrm{C}$ for $30 \mathrm{~min}$. Tissue sections were dewaxed at room temperatures as follows: xylene I (100\%) for $15 \mathrm{~min}$, xylene II $(100 \%)$ for $15 \mathrm{~min}$, and then a graded series of ethanol $(100,95,80$ and $70 \%)$ for 5 min each. To uncover the antigens sufficiently, the sections were submerged for antigenic retrieval in citrate buffer (for AGR3, pH 6.0) and EDTA buffer (for TP53, $\mathrm{pH} 9.0$ ) at $96-99^{\circ} \mathrm{C}$ for $15 \mathrm{~min}$, then cooled to room temperature. The activity of endogenous peroxidases was inhibited with $3 \%$ hydrogen peroxide at $37^{\circ} \mathrm{C}$ for $15 \mathrm{~min}$. Subsequently, the non-specific binding sites were blocked with goat serum from the IHC reagent kit (cat. no. SP9000; OriGene Technologies, Inc., Beijing, China). The mouse anti-human monoclonal antibody for AGR3 (cat. no. ab82400; Abcam) was applied at a dilution of 1:4,000 and mouse anti-human monoclonal antibody for TP53 (cat. no. ZM0408; OriGene Technologies, Inc.) at the working concentration. Then, the slides were stored in a moist chamber at room temperature for $2 \mathrm{~h}$. Following washing of the sections (PBS, 3 times for $3 \mathrm{~min}$ ), they were incubated with the secondary antibody from IHC reagent kit (OriGene Technologies, Inc.) at $37^{\circ} \mathrm{C}$ for $20 \mathrm{~min}$, and then the sections were washed again as above. Sections were stained with diaminobenzidine for $1.5 \mathrm{~min}$ at room temperature and counterstained with hematoxylin $(0.2 \%)$ for $2 \mathrm{~min}$ at room temperature. Negative controls were made by omitting the primary antibody. A light microscope (Nikon Corporation, Tokyo, Japan) was used to visualize the stained tissue sections.

AGR3 was scored as positive or negative and TP53 was scored as aberrant expression or wild-type pattern. The subcellular localization of AGR3 on staining was predominantly found to be membranous, whereas for TP53 it was nuclear. More than or equal to $20 \%$ positively stained in epithelial cells were defined as positive for AGR3. While aberrant expression of TP53 was defined as 0 (negative or occasional cells positive) or $\geq 75 \%$ of cells staining, according to clinical practice and previous studies (22), and also because of evidence that these TP53-staining patterns correlated with the mutational status of TP53 $(23,24)$. Thus, absence and overexpression of TP53 were considered as aberrant expression, indicating various types of TP53 mutation. The usefulness of TP53 and AGR3 to distinguish HGSC and LGSC was evaluated with a $\chi^{2}$ test. Then, the TP53/AGR3 differential diagnostic performance was quantified with sensitivity and specificity calculations with morphological classification as the gold standard. Sensitivity, also termed the true positive rate, is defined as the proportion of positives that are correctly identified as such. Specificity, also termed the true negative rate, is defined as the proportion of negatives that are correctly identified as such. In order to evaluate the reliability of the diagnostic test, positive and negative predictive value were calculated. The positive and negative predictive values (PPV and NPV, respectively) are defined as the proportions of positive and negative results in diagnostic tests that are true positive and true negative results, respectively. Receiver operating characteristic (ROC) analysis was performed to compare diagnostic effectiveness of TP53 and AGR3. Then, unsupervised clustering analysis of TP53 and AGR3 IHC scores was performed using heatmap.2 function in $\mathrm{R}$ (version 3.4.2). Based on clustering results, combination of TP53 and AGR3 data were tabulated to distinguish HGSC and LGSC. All statistical analyses were performed using SPSS 22.0 (IBM Corp., Armonk, NY, USA).

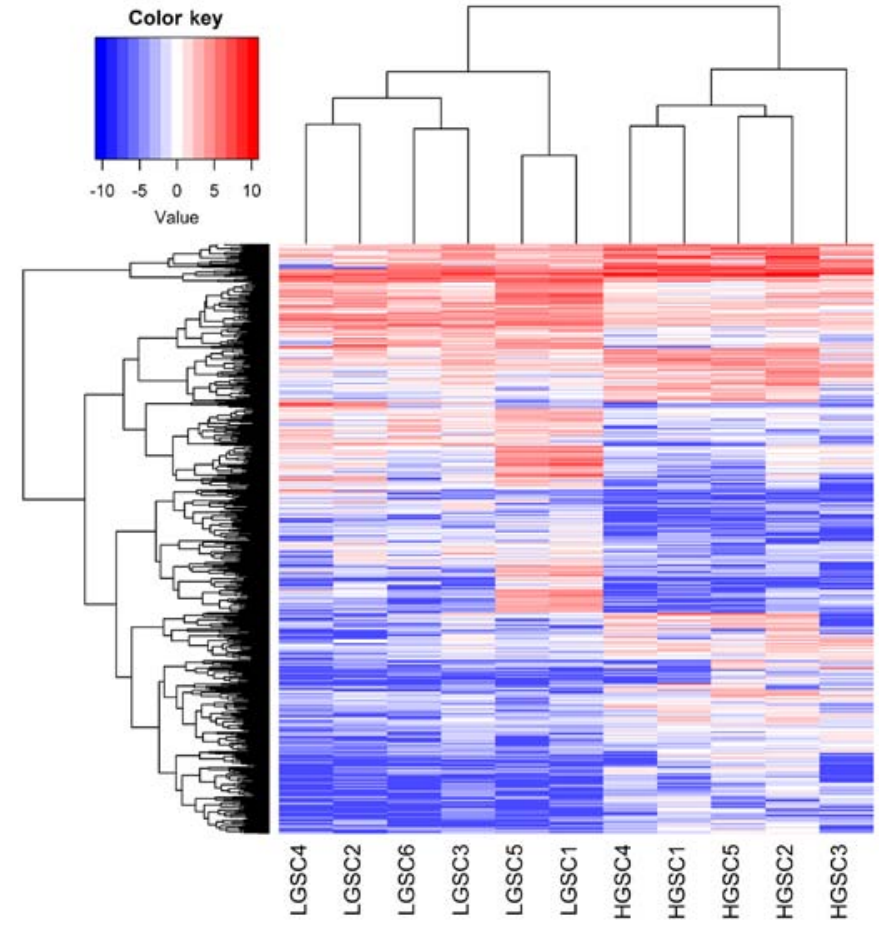

Figure 1. Dendrograms produced by unsupervised hierarchical clustering of high-grade $(n=5)$ and low-grade $(n=6)$ serous carcinomas. Unsupervised hierarchical cluster analyses on differentially expressed genes separated the 11 samples into two distinct groups. HGSC, high-grade serous carcinoma; LGSC, low-grade serous carcinoma.

Statistical analysis. HTSeq (v0.9.1; open-source software available from www-huber.embl.de/HTSeq or from the Python Package Index at pypi.python.org/pypi/HTSeq) was used to analyze RNA-seq data. Differential expression analysis between HGSC and LGSC was performed using the DESeq R package (version 3.4.2; open-source available from www.r-project.org/). Data achieved from RT-qPCR are presented as the mean \pm standard deviation and analyzed through t-test using GraphPad Prism (version 5.01; GraphPad Software, Inc., La Jolla, CA, USA) software. $\chi^{2}$ test was performed for IHC analysis using SPSS 19.0 software (IBM Corp.). $\mathrm{P}<0.05$ was considered to indicate a statistically significant difference.

\section{Results}

HGSC and LGSC are two distinct diseases with different gene expression profiles. Comparing HGSC and LGSC using the DESeq R package, 699 differentially expressed genes ( $>2$-fold difference) were identified, 367 of which were upregulated in HGSC. Unsupervised hierarchical cluster analyses on differentially expressed genes identified by mRNA sequencing between HGSC and LGSC demonstrated that HGSC clearly segregated from LGSC (Fig. 1). A number of differentially expressed genes between them were in accordance with previous studies (14). According to the Gene Ontology analysis, significant groups of differential genes between HGSC and LGSC associated with specific functional processes, including 'pattern specification process', 'DNA metabolic process' and 'nucleic acid metabolic process'. 
A

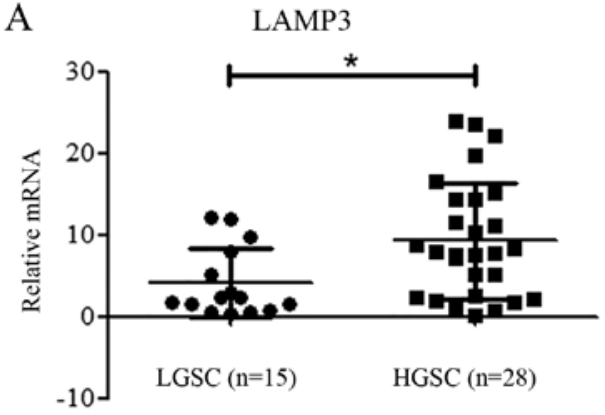

$\mathrm{C}$

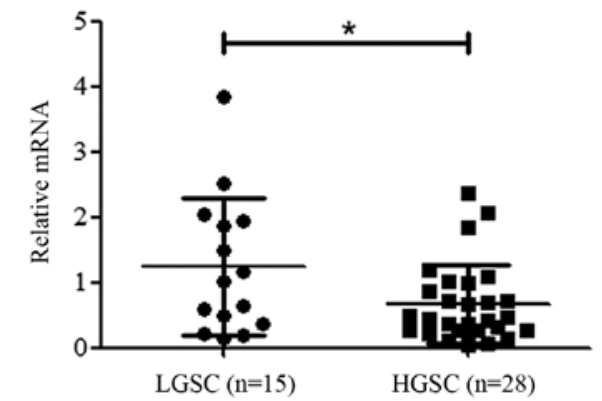

B

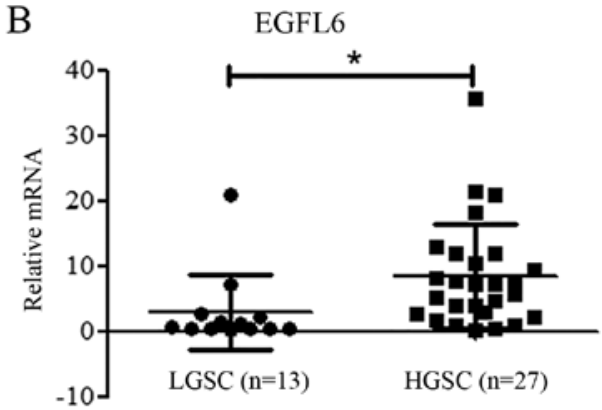

$\mathrm{D}$

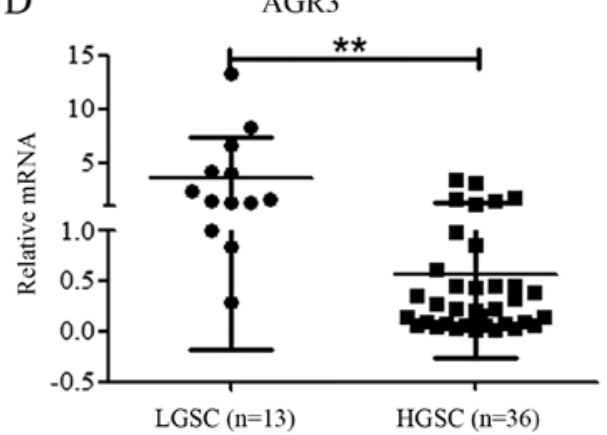

Figure 2. Expression of LAMP3, EGFL6, CDKN1A and AGR3 in HGSC and LGSC. RT-qPCR confirmed a trend toward increased expression of (A) LAMP3 and (B) EGFL6 in HGSC samples compared with LGSC ( $\mathrm{P}=0.0137$ and $\mathrm{P}=0.0365$ respectively). RT-qPCR confirmed the decreased expression of (C) CDKN1A and (D) AGR3 in HGSC samples compared with LGSC ( $\mathrm{P}=0.0285$ and $\mathrm{P}<0.001$ respectively). ${ }^{*} \mathrm{P}<0.05$, ${ }^{* *} \mathrm{P}<0.01$. RT-qPCR, reverse transcription-quantitative polymerase chain reaction; HGSC, high-grade serous carcinoma; LGSC, low-grade serous carcinoma; LAMP3, lysosomal associated membrane protein 3; EGFL6, EGF like domain multiple 6; CDKN1A, cyclin-dependent kinase inhibitor 1A; AGR3, anterior gradient homolog 3.

To validate the results and to further investigate novel differential diagnostic markers, certain differential genes were analyzed by RT-qPCR. As shown in Fig. 2, LAMP3 and EGFL6 were upregulated in HGSC, while CDKN1A and AGR3 were significantly downregulated compared with LGSC. These results, in accordance with mRNA sequencing results, indicate that gene expression profile in HGSC is different from that in LGSC. Among these genes, AGR3, which exhibited the greatest difference between HGSC and LGSC was selected for further analysis.

Significant differential expression of TP53 and AGR3 protein in HGSC and LGSC. Previous researches have confirmed the use of TP53 in the differential diagnosis of HGSC and LGSC. On this basis, the diagnostic utility of AGR3 alone and combined with TP53 were investigated in the current study. The protein expression of TP53 and AGR3 in HGSC $(n=8)$ and LGSC $(n=7)$ were examined through western blot analysis. TP53 exhibited overexpression or absence in 6 of 8 HGSC compared with LGSC, as expected. Increased expression of AGR3 was observed in LGSC compared with HGSC samples (Fig. 3). Taken together, these findings suggest that the differential expression of TP53 and AGR3 between HGSC and LGSC may serve as markers for differential diagnosis.

TP53 and AGR3 were useful to distinguish HGSC from $L G S C$. In order to assess the diagnostic performance of TP53 and AGR3, immunohistochemical staining was performed on 145 HGSC and 30 LGSC samples to analyze sensitivity and specificity of these markers in diagnosis. The subcellular

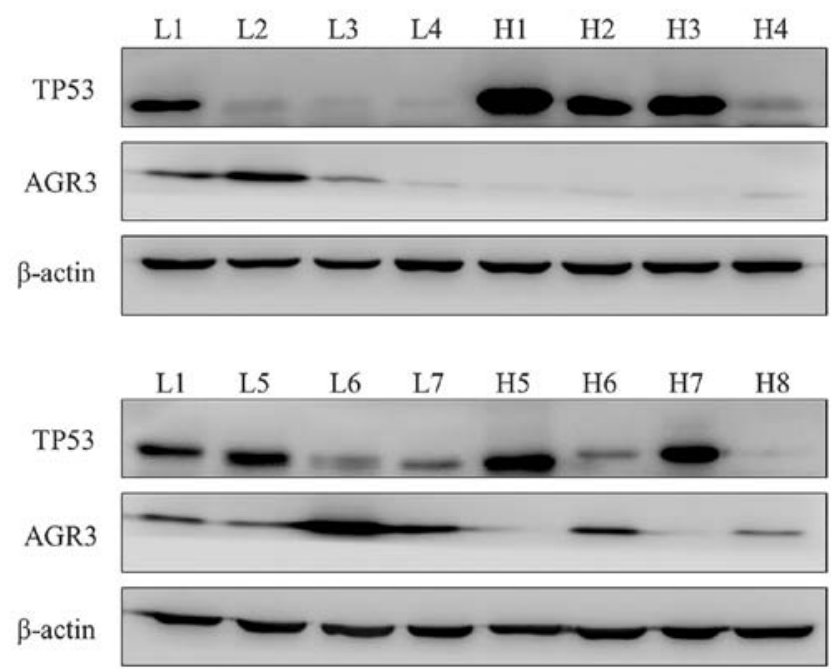

Figure 3. Expression of TP53 and AGR3 protein in HGSC and LGSC. $\mathrm{H}$ and $\mathrm{L}$ represent HGSC and LGSC, respectively. $\mathrm{L} 1$ is set as repeat sample between the two blots for comparison. Protein level of TP53 is higher in HGSC than LGSC. Protein level of AGR3 is lower in HGSC than LGSC. HGSC, high-grade serous carcinoma; LGSC, low-grade serous carcinoma; TP53, tumor protein 53; AGR3, anterior gradient homolog 3.

localization of TP53 staining was predominantly nuclear. Additionally, the percentage of positive cells, rather than immunointensity, was used for evaluation of TP53 in the majority of previous studies $(22,25)$. With respect to AGR3, its subcellular localization was predominantly membranous. There was no different immunointensity between two samples with sharp distinction in positive cell percentage. Thus, the percentage of 

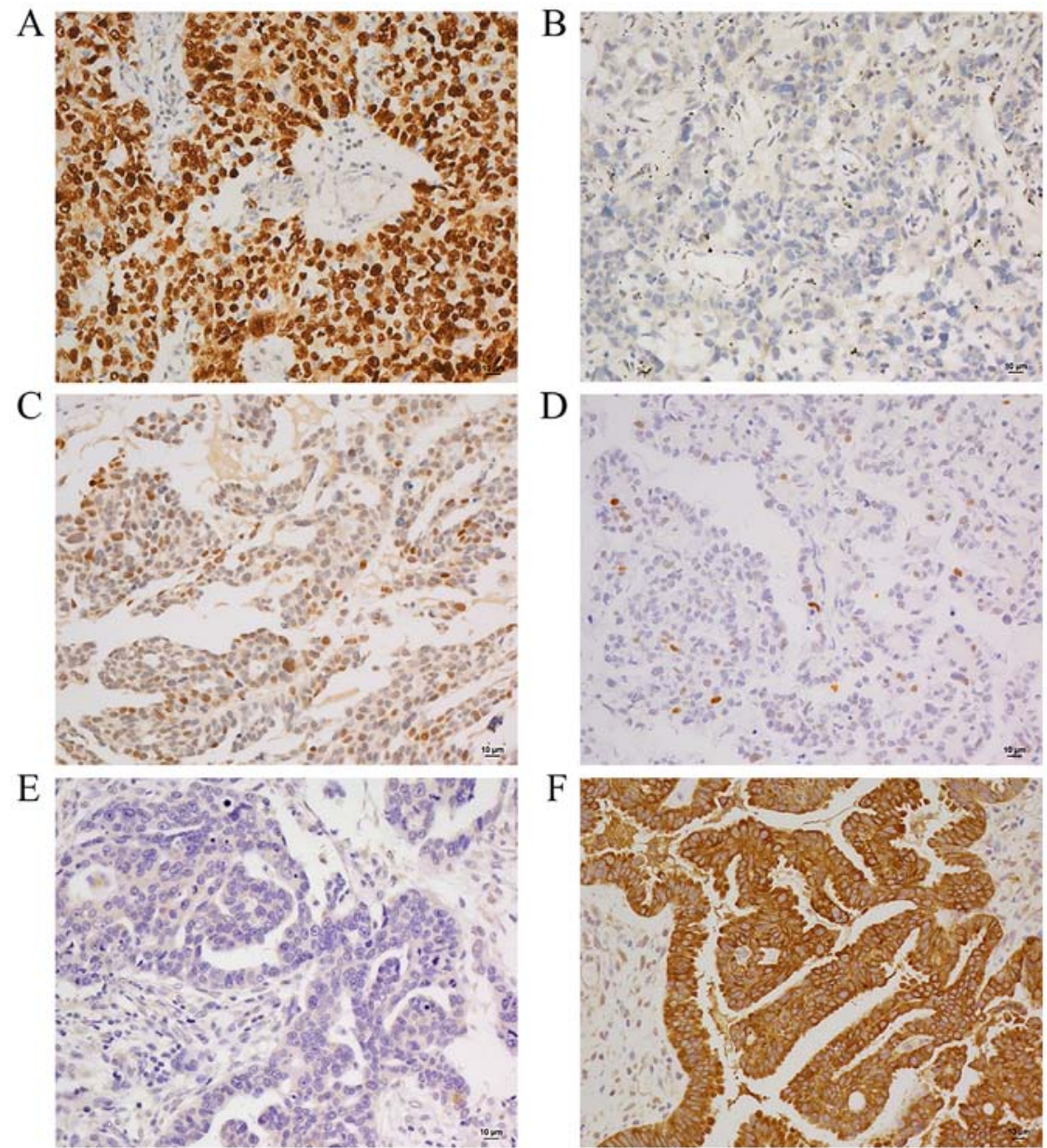

Figure 4. Representative stains of TP53 and AGR3 in HGSC and LGSC. (A) HGSC with diffuse TP53 staining. (B) HGSC with complete absence of TP53 staining. (C) HGSC with focal TP53 staining. (D) LGSC with focal TP53 staining. (E) HGSC with negative AGR3 expression. (F) LGSC with positive AGR3 expression. Magnification, x200. Scale bar, $10 \mu \mathrm{m}$. HGSC, high-grade serous carcinoma; LGSC, low-grade serous carcinoma; TP53, tumor protein 53; AGR3, anterior gradient homolog 3 .

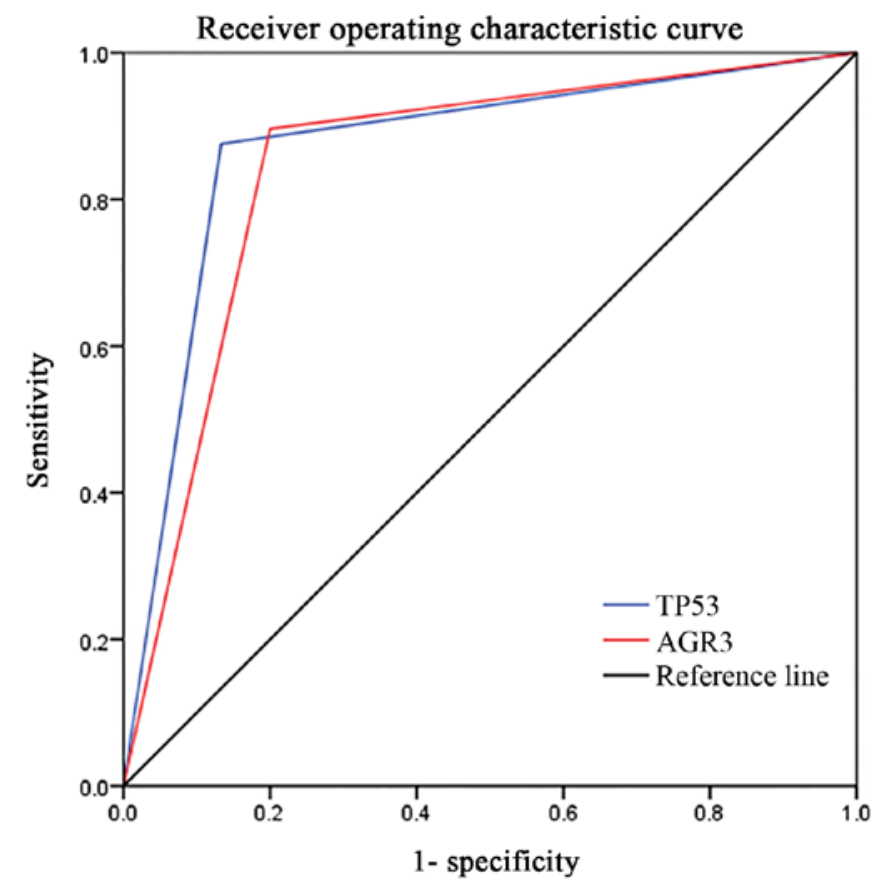

Figure 5. Receiver operating characteristic curve analysis of TP53 and AGR3. Area under the curve: 0.871 for TP53 and 0.848 for AGR3. TP53, tumor protein 53; AGR3, anterior gradient homolog 3. positive cells was used for evaluation of TP53 and AGR3, as in previous studies $(25,26)$. Representative staining features of TP53 and AGR3 in HGSC and LGSC are shown in Fig. 4. TP53 was scored as absent (negative or occasional positive cells), wild-type pattern (0-75\%) or overexpression ( $\geq 75 \%)$ and AGR3 was scored as either negative $(<20 \%)$ or positive $(\geq 20 \%)$. The staining results for TP53 and AGR3 across the available cohorts were shown in Tables II and III. TP53 and AGR3 were efficient in distinguishing HGSC from LGSC ( $\mathrm{P}<0.001$ for both). To test the diagnostic reliability of TP53 and AGR3, positive and negative predictive values were also calculated in the same samples again. Aberrant TP53 staining ( 0 or $\geq 75 \%$ positive staining) was detected in $127 / 145$ (87.6\%) of HGSC and in 4/30 (13.3\%) of LGSC samples. The positive staining of AGR3 in HGSC and LGSC was 15/145 (11.3\%) and 24/30 (80.0\%) respectively. ROC analysis produced an area under the curve of 0.871 for TP53, and 0.848 for AGR3 (Fig. 5). Thus, AGR3 was also a useful marker with respect to differential diagnosis. PPV and NPV were 96.9 and 59.1\% for TP53, and 61.5 and $95.6 \%$ for AGR3, which reminds us to combine the two markers for an overall analysis.

In order to evaluate the diagnostic performance of a combination of TP53 and AGR3 staining, a heat map was produced by unsupervised hierarchical cluster analysis in $\mathrm{R}$. 


\begin{tabular}{|c|c|c|c|}
\hline Cluster & Predicted type & Accuracy & Staining pattern \\
\hline 1 & $\begin{array}{l}\text { High-grade serous } \\
\text { carcinoma }\end{array}$ & $99.1 \%$ & TP53 abnormal expression/AGR3-negative \\
\hline 2 & $\begin{array}{l}\text { High-grade serous } \\
\text { carcinoma }\end{array}$ & $80.0 \%$ & TP53 abnormal expression/AGR3-positive \\
\hline 3 & $\begin{array}{l}\text { Low-grade serous } \\
\text { carcinoma }\end{array}$ & $87.5 \%$ & TP53 wild-type/AGR3-positive \\
\hline 4 & $\begin{array}{l}\text { High-grade serous } \\
\text { carcinoma }\end{array}$ & $75.0 \%$ & TP53 wild-type/AGR3-negative \\
\hline
\end{tabular}

Figure 6. Heat map produced by unsupervised hierarchical cluster analysis. Left column, black represents TP53 and orange represent AGR3. Right column, red and blue represent staining pattern of TP53 and AGR3. Four possible cluster groups were shown in heat map. TP53, tumor protein 53; AGR3, anterior gradient homolog 3 .

Table II. Test performance of TP53 for diagnosis of HGSC.

\begin{tabular}{lrrr}
\hline TP53 expression & HGSC & LGSC & P-value \\
\hline Abnormal & 127 & 4 & $<0.001$ \\
Normal & 18 & 26 & \\
Total & 145 & 30 &
\end{tabular}

Sensitivity, $87.6 \%$; specificity, $86.7 \%$. Positive predictive value, $96.9 \%$; negative predictive value, $59.1 \%$. P-value was generated using $\chi^{2}$ test. TP53 abnormal expression, 0 (negative or occasional cells positive) or $\geq 75 \%$ positive expression; TP53 wild-type, 0 - 75 positive expression. HGSC, high-grade serous carcinoma; LGSC, low-grade serous carcinoma; TP53, tumor protein 53.

Table III. Test performance of AGR3 for diagnosis of LGSC.

\begin{tabular}{lrrrr}
\hline AGR3 expression & HGSC & LGSC & Total & P-value \\
\hline Positive & 15 & 24 & 39 & $<0.001$ \\
Negative & 130 & 6 & 136 & \\
Total & 145 & 30 & 175 & \\
\hline
\end{tabular}

Specificity, $89.7 \%$; sensitivity, $80.0 \%$.Positive predictive value, $61.5 \%$; negative predictive value, $95.6 \%$. P-value was generated using $\chi^{2}$ test. AGR3-positive, $\geq 20 \%$ positive expression; AGR3-negative, $<20 \%$ positive expression. HGSC, high-grade serous carcinoma; LGSC, low-grade serous carcinoma; AGR3, anterior gradient homolog 3 .

With two markers (TP53 and AGR3, each with two outcomes), four combinations/cluster groups were derived (Fig. 6). Three of these four cluster groups were associated with HGSC.
Table IV. Test performance of cluster 3 for diagnosis of LGSC.

\begin{tabular}{lcrc}
\hline Cluster & HGSC & LGSC & P-value \\
\hline 3 & 3 & 21 & $<0.001$ \\
$1,2,4$ & 142 & 9 & \\
Total & 145 & 30 & \\
\hline
\end{tabular}

Specificity, $97.9 \%$; sensitivity, $70 \%$. Positive predictive value, $87.5 \%$; negative predictive value, $94.0 \%$. P-value was generated using $\chi^{2}$ test. HGSC, high-grade serous carcinoma; LGSC, low-grade serous carcinoma.

Cluster 1 (TP53 aberrant expression and AGR3-negative), accounting for $66.3 \%$ of the cases, $99.1 \%$ were diagnosed as HGSC by the gold standard morphology. Cluster 2 (TP53 aberrant expression and AGR3-positive), which formed the smallest group with $8.6 \%$ of the cases, was associated with the diagnosis of HGSC. Cluster 4 (TP53 wild-type staining and AGR3-negative) was also most likely associated with HGSC. However, a substantial proportion of LGSC was found in cluster 3 (TP53 wild-type staining and AGR3-positive).

Given that only cluster 3 is predictive for LGSC, the performance of cluster 3 was analyzed for use in diagnosis of LGSC with sensitivity and specificity shown in Table IV. The sensitivity of cluster 3 to predict LGSC is $70.0 \%$ (21/30) with a specificity of $97.9 \%(142 / 145)$. In order to test the predictive performance for clinical diagnosis, PPV and NPV were calculated using the current samples. PPV and NPV of cluster 3 were 87.5 and $94.0 \%$, respectively. In addition, the accuracy of differential diagnosis was $93.1 \%$ (163/175). These results suggest that a combination of TP53 and AGR3 may be highly effective in distinguishing HGSC from LGSC. 


\section{Discussion}

HGSCs and LGSCs exhibit distinct characteristics, and possess diverse pathogenesis and prognosis. HGSC is highly aggressive, grows rapidly and almost always presents at an advanced stage. It directly develops from serous tubal intraepithelial carcinoma (27). According to The Cancer Genome Atlas ovarian cancer study, TP53 mutation occurs in $96 \%$ of HGSCs, while mutations of KRAS proto-oncogene, GTPase (KRAS), B-Raf proto-oncogene, serine/threonine kinase (BRAF) or (erb-b2 receptor tyrosine kinase 2) ERBB2 are infrequent (28). LGSC accounts for a smaller proportion of all ovarian serous carcinomas. LGSC generally exhibits indolent biological behavior and is usually confined to the ovary at presentation. It has been hypothesized to develop in a slow step-wise manner from a serous cystadenoma or adenofibroma (29-31). LGSC is relatively stable and expresses normal levels of TP53, but also is characterized by mutations of the KRAS, BRAF or ERBB2 genes (8). Furthermore, HGSC and LGSC respond differentially to similar therapeutic protocols, so it is crucial to distinguish HGSC from LGSC prior to the initiation of treatment. The final diagnosis relies on histopathological features, however clinical behavior, ancillary examinations, molecular biology characteristics and especially immunohistochemical markers are all useful in differentiating HGSC from LGSC. Currently, a two-tier system is widely used to subdivide ovarian serous carcinoma into HGSC and LGSC. Excellent inter- and intraobserver reproducibility of the two-tier system for grading ovarian serous carcinoma has been demonstrated. However, certain difficulties remain in differential diagnosis of HGSC and LGSC. On one hand, certain tumors with intermediate characteristics cannot be categorized accurately even with the application of the two-tier grading scheme, especially in local hospitals. On the other hand, current clinical practice can only rely on limited samples from paracentesis or biopsies of pelvic/peritoneal implants prior to the commencement of neoadjuvant chemotherapy or targeted therapy. Under such conditions, a number of markers (including P16, Ki67 and TP53) are investigated for differential diagnosis of ovarian serous carcinoma $(22,32)$. In a previous study, the expression of P16 was analyzed in HGSC and LGSC. The cases were semi-quantitatively scored as 0 (negative or occasional positive cells), $1+(<10 \%$ cells positive), $2+(10-25 \%$ cells positive), $3+$ ( $26-50 \%$ cells positive), $4+(51-75 \%$ cells positive) or $5+(>75 \%$ cells positive). In the previous study, $5+$ staining ( $>75 \%$ positive cells) was observed in $27 \%$ of LGSC and in $83 \%$ of HGSC (32). A diffusely positive staining pattern (5+ staining) may result into an erroneous diagnosis because many metastatic ovarian tumors arising from uterine serous carcinomas also exhibit the same staining pattern for P16. Similarly, the Ki67 proliferation index and the expression of TP53 are both higher in HGSC compared with LGSC. In a study by $\mathrm{O}^{\prime} \mathrm{Neill}$ et al (22), the proliferation index was significantly different between these two tumor types (23\% for low-grade and 55\% for high-grade). TP53 5+ staining (>75\% positive cells) was observed in $18 \%$ of LGSC and $64 \%$ of HGSC (22). Additionally, another study reported that aberrant expression of TP53 (completely absent or $\geq 60 \%$ of cells stained) occurred in $89 \%$ of HGSC and $6 \%$ of LGSC (25).
These studies indicate that HGSC is characterized by diffuse expression of TP53, while the expression of TP53 is much lower in LGSC. To the best of our knowledge, molecular alteration of TP53 is a defining feature of HGSC and TP53 is also currently one of the best available choices for differential diagnosis (33). However, TP53 staining does not perfectly differentiate HGSC from LGSC on the basis of morphology. Thus, we intend to identify novel and effective markers to be used in consolidation with TP53 in order to distinguish HGSC from LGSC efficiently on the basis of conventional histopathology.

In total, 699 differentially expressed genes were identified comparing HGSC and LGSC using DESeq R package and dendrograms were produced by unsupervised hierarchical clustering using heatmap. 2 function in 'gplots' R-package. HGSC was separated from LGSC completely. The results support the hypothesis of two distinct pathways of HGSC and LGSC yielding two different disease entities. This is in accordance with the different clinical and molecular presentations of the two. In agreement with the sequencing data, HGSC expressed higher levels of LAMP3 and EGFL6 mRNA compared with LGSC, as determined by RT-qPCR $(\mathrm{P}<0.05)$. While the mRNA levels of CDKN1A and AGR3 were higher in LGSC $(\mathrm{P}<0.05)$. AGR3, which exhibited the greatest variation out of the four molecules between HGSC and LGSC, was selected for further analysis. TP53 was included in the current study based on its diagnostic utility. Along with mRNA levels, AGR3 protein expression was also higher in LGSC, as demonstrated using western blot analysis. The findings suggested that AGR3 may be used as an efficient marker and may be beneficial in differential diagnosis between HGSC and LGSC. However, the comparison of TP53 expression between HGSC and LGSC using western blotting was solely circumstantial evidence. Regrettably, mutation data on HGSC and LGSC was not analyzed. Furthermore, overexpression of TP53 demonstrated by western blotting cannot rule in or rule out the role of TP53 mutations.

In the current study, aberrant expression of TP53 ( 0 or $\geq 75 \%$ positive) exhibited a sensitivity $87.6 \%$ and specificity $86.7 \%$ for HGSC. Overexpression and absence of TP53 in HGSC indicates missense and null mutations, respectively. Although, it is generally acknowledged that vast majority of HGSCs are characterized by TP53 mutations and numerous studies have reported that TP53 staining patterns were distinctly different between HGSC and LGSC $(25,34)$. However, based on a study by Singer et al (35), immunohistochemistry does not accurately predict mutation status. Furthermore, TP53 staining does not perfectly differentiate HGSC from LGSC. Therefore, it is necessary to seek additional markers to improve the current diagnostic efficacy.

The differential diagnostic value of AGR3 for HGSC and LGSC were investigated. To the best of our knowledge, there are only limited reports of AGR3 expression in normal and cancer tissue. The current study confirmed that the expression of AGR3 was markedly different between HGSC and LGSC. Following analysis of the expression with varied cutoff values, $20 \%$ AGR3-positive cells was determined to be the best cut-off to distinguish HGSC from LGSC. Therefore, $20 \%$ or more positively stained epithelial cells was defined as AGR3-positive. Positive expression of AGR3 has a sensitivity of $80.0 \%$ and a 
specificity of $89.7 \%$ for LGSC. Although there is some overlap in the extent of staining in HGSC vs. LGSC, these findings indicate that a morphologically problematic serous ovarian carcinoma with negative expression of AGR3 is unlikely to be LGSC. ROC analysis produced a similar area under the curve for AGR3 compared to TP53, suggesting that AGR3 is comparably as important as TP53 for differential diagnosis of HGSC and LGSC. PPV and NPV of AGR3 staining for LGSC were 61.5 and $95.6 \%$, respectively. The low PPV may be due to the finite number of LGSC samples. In accordance, the expression of AGR3 was also reported to be significantly higher in LGSC than HGSC by western blot analysis in a previous study by King et al (26). However, the previous study could not distinguish HGSC from LGSC using AGR3 IHC, which was likely attributable to the different anti-AGR3 antibody used and different cutoff values in the two studies. In the current study, $20 \%$ was the best cutoff to efficiently distinguish HGSC from LGSC. The results support the association of AGR3 with the differentiation of serous ovarian cancer. In conclusion, AGR3 is a useful marker for differential diagnosis of HGSC and LGSC based on the results of the present study. Subsequently, statistical validation was performed for combined application of AGR3 and TP53 in the differential diagnosis of HGSC and LGSC. The strength of the study is depicted by the PPV $(87.5 \%)$ and NPV (94.0\%). Furthermore, the accuracy of diagnosis increased from $87.4 \%$ to $93.1 \%$ by including AGR3. Thus, a panel of two antibodies is more effective and it improves the accuracy of the diagnosis. IHC staining with a wild-type TP53 pattern and AGR3-positive expression produced a specificity of $97.9 \%$ and a sensitivity of $70.0 \%$ for LGSC. The low sensitivity may be attributed to a proportion of samples with negative expression of AGR3 and aberrant expression of TP53 in LGSC. The outcomes ascertained from the current study provide compelling evidence for the use of these two markers for the differential diagnosis of HGSC and LGSC.

In summary, these results suggest that a combination of TP53 and AGR3 staining is superior to TP53 alone for differential diagnosis of HGSC and LGSC on the basis of morphology. Furthermore, it may improve accuracy of diagnosis and be beneficial for the clinical management, optimizing therapeutic regimen and thus lowering the overall risk of mortality. On this basis, the two markers may be useful to test additional ovarian carcinomas of 'uncertain' subtype. However, there is no statistical data at present because of limitation in the number of rare cases. Future prospective clinical studies should be conducted on a larger cohort of various ovarian carcinoma subtypes to validate the clinical utility of the model.

\section{Acknowledgements}

Not applicable.

\section{Funding}

This study was supported by National Natural Science Foundation of China (grant nos. 81572554 and 81272857), the National Clinical Research Center for Gynecological Oncology (grant no. 2015BAI13B05) and the Natural Science Foundation of Shandong Province (grant nos. ZR2014HM070 and ZR2015HM079).

\section{Availability of data and materials}

The datasets used and/or analyzed during the current study are available from the corresponding author on reasonable request.

\section{Authors' contributions}

BK designed and supervised the research. NL provided technical support and revised the paper. CQ was a major contributor in collecting tissue samples and writing the manuscript. YW performed statistical analysis. XW reviewed the sections used for further study and interpreted all data from immunohistochemistry. QZ collected and analyzed RNA-seq experiments. YL carried out RT-qPCR analysis. YX performed western blotting. CJ and HB conducted all immunohistochemistry experiments. WZ reviewed and confirmed the sections used for further study. XY directed the research. All authors have read and approved the final manuscript.

\section{Ethics approval and consent to participate}

Samples were collected with the approval of the Ethics Committee at Qilu Hospital of Shandong University (Jinan, China), and with the signed informed consent from all patients.

\section{Consent for publication}

Not applicable.

\section{Competing interests}

The authors declare that they have no competing interests.

\section{References}

1. Siegel RL, Miller KD and Jemal A: Cancer Statistics, 2017. CA Cancer J Clin 67: 7-30, 2017.

2. Prat J: Ovarian carcinomas: Five distinct diseases with different origins, genetic alterations, and clinicopathological features. Virchows Archiv 460: 237-249, 2012.

3. Köbel M, Kalloger SE, Boyd N, McKinney S, Mehl E, Palmer C, Leung S, Bowen NJ, Ionescu DN, Rajput A, et al: Ovarian carcinoma subtypes are different diseases: Implications for biomarker studies. PLoS Med 5: e232, 2008.

4. Malpica A, Deavers MT, Lu K, Bodurka DC, Atkinson EN, Gershenson DM and Silva EG: Grading ovarian serous carcinoma using a two-tier system. Am J Surg Pathol 28: 496-504, 2004

5. Bertelsen K, Holund B and Andersen E: Reproducibility and prognostic value of histologic type and grade in early epithelial ovarian cancer. Int J Gynecol Cancer 3: 72-79, 1993.

6. Shimizu Y, Kamoi S, Amada S, Hasumi K, Akiyama F and Silverberg SG: Toward the development of a universal grading system for ovarian epithelial carcinoma. I. Prognostic significance of histopathologic features - problems involved in the architectural grading system. Gynecol Oncol 70: 2-12, 1998.

7. Silverberg SG: Histopathologic grading of ovarian carcinoma: A review and proposal. Int J Gynecol Pathol 19: 7-15, 2000.

8. Vang R, Shih IeM and Kurman RJ: Ovarian low-grade and highgrade serous carcinoma: Pathogenesis, clinicopathologic and molecular biologic features, and diagnostic problems. Adv Anat Pathol 16: 267-282, 2009.

9. Malpica A, Deavers MT,Lu K, Liu J, Atkinson EN, Gershenson DM and Silva EG: Grading ovarian serous carcinomas using a two-tier system. Mod Pathol 15: 202a-203a, 2002.

10. Tone AA, Begley H, Sharma M, Murphy J, Rosen B, Brown TJ and Shaw PA: Gene expression profiles of luteal phase fallopian tube epithelium from BRCA mutation carriers resemble high-grade serous carcinoma. Clin Cancer Res 14: 4067-4078, 2008. 
11. Marquez RT, Baggerly KA, Patterson AP, Liu J, Broaddus R, Frumovitz M, Atkinson EN, Smith DI, Hartmann L, Fishman D, et al: Patterns of gene expression in different histotypes of epithelial ovarian cancer correlate with those in normal fallopian tube, endometrium, and colon. Clin Cancer Res 11: 6116-6126, 2005.

12. Li J, Abushahin N, Pang S, Xiang L, Chambers SK, Fadare O, Kong B and Zheng W: Tubal origin of 'ovarian' low-grade serous carcinoma. Mod Pathol 24: 1488-1499, 2011.

13. Qiu C, Lu N, Wang X, Zhang Q, Yuan C, Yan S, Dongol S, Li Y, Sun X, Sun C, et al: Gene expression profiles of ovarian low-grade serous carcinoma resemble those of fallopian tube epithelium. Gynecol Oncol 147: 634-641, 2017.

14. May T, Shoni M, Crum CP, Xian W, Vathipadiekal V, Birrer M, Rosen B, Tone A and Murphy KJ: Low-grade and high-grade serous Mullerian carcinoma: Review and analysis of publicly available gene expression profiles. Gynecol Oncol 128: 488-492, 2013.

15. Vergote I, Tropé CG, Amant F, Kristensen GB, Ehlen T, Johnson N, Verheijen RHM, van der Burg MEL, Lacave AJ, Panici PB, et al; European Organization for Research and Treatment of Cancer-Gynaecological Cancer Group; NCIC Clinical Trials Group: Neoadjuvant chemotherapy or primary surgery in stage IIIC or IV ovarian cancer. N Engl J Med 363 943-953, 2010 .

16. Pohl G, Ho CL, Kurman RJ, Bristow R, Wang TL and Shih IeM: Inactivation of the mitogen-activated protein kinase pathway as a potential target-based therapy in ovarian serous tumors with KRAS or BRAF mutations. Cancer Res 65: 1994-2000, 2005.

17. Malpica A, Deavers MT, Tornos C, Kurman RJ, Soslow R, Seidman JD, Munsell MF, Gaertner E, Frishberg D and Silva EG: Interobserver and intraobserver variability of a two-tier system for grading ovarian serous carcinoma. Am J Surg Pathol 31: 1168-1174, 2007.

18. Ayhan A, Kurman RJ, Yemelyanova A, Vang R, Logani S, Seidman JD and Shih IeM: Defining the cut point between low-grade and high-grade ovarian serous carcinomas: A clinicopathologic and molecular genetic analysis. Am J Surg Pathol 33 1220-1224, 2009.

19. Kalloger SE, Köbel M, Leung S, Mehl E, Gao D, Marcon KM, Chow C, Clarke BA, Huntsman DG and Gilks CB: Calculator for ovarian carcinoma subtype prediction. Mod Pathol 24: 512-521, 2011.

20. Kim D, Pertea G, Trapnell C, Pimentel H, Kelley R and Salzberg SL: TopHat2: Accurate alignment of transcriptomes in the presence of insertions, deletions and gene fusions. Genome Biol 14: R36, 2013.

21. Mortazavi A, Williams BA, McCue K, Schaeffer L and Wold B: Mapping and quantifying mammalian transcriptomes by RNA-Seq. Nat Methods 5: 621-628, 2008.

22. O'NeillCJ,Deavers MT,Malpica A,FosterH and McCluggage WG: An immunohistochemical comparison between low-grade and high-grade ovarian serous carcinomas: Significantly higher expression of p53, MIB1, BCL2, HER-2/neu, and C-KIT in highgrade neoplasms. Am J Surg Pathol 29: 1034-1041, 2005.

23. Yemelyanova A, Vang R, Kshirsagar M, Lu D, Marks MA, Shih IeM and Kurman RJ: Immunohistochemical staining patterns of p53 can serve as a surrogate marker for TP53 mutations in ovarian carcinoma: An immunohistochemical and nucleotide sequencing analysis. Mod Pathol 24: 1248-1253, 2011.
24. Cole AJ, Dwight T, Gill AJ, Dickson KA, Zhu Y, Clarkson A Gard GB, Maidens J, Valmadre S, Clifton-Bligh R, et al: Assessing mutant p53 in primary high-grade serous ovarian cancer using immunohistochemistry and massively parallel sequencing. Sci Rep 6: 26191, 2016.

25. Altman AD, Nelson GS, Ghatage P, McIntyre JB, Capper D, Chu P, Nation JG, Karnezis AN, Han G, Kalloger SE, et al: The diagnostic utility of TP53 and CDKN2A to distinguish ovarian high-grade serous carcinoma from low-grade serous ovarian tumors. Mod Pathol 26: 1255-1263, 2013.

26. King ER, Tung CS, Tsang YTM, Zu Z, Lok GTM, Deavers MT, Malpica A, Wolf JK, Lu KH, Birrer MJ, et al: The anterior gradient homolog 3 (AGR3) gene is associated with differentiation and survival in ovarian cancer. Am J Surg Pathol 35: 904-912, 2011.

27. Vang R, Shih IeM and Kurman RJ: Fallopian tube precursors of ovarian low- and high-grade serous neoplasms. Histopathology 62: 44-58, 2013.

28. Bell D, Berchuck A, Birrer M, Chien J, Cramer DW, Dao F, Dhir R, DiSaia P, Gabra H, Glenn P, et al; Cancer Genome Atlas Research Network: Integrated genomic analyses of ovarian carcinoma. Nature 474: 609-615, 2011.

29. Smith Sehdev AE, Sehdev PS and Kurman RJ: Noninvasive and invasive micropapillary (low-grade) serous carcinoma of the ovary: A clinicopathologic analysis of 135 cases. Am J Surg Pathol 27: 725-736, 2003.

30. Bell DA, Longacre TA, Prat J, Kohn EC, Soslow RA, Ellenson LH, Malpica A, Stoler MH and Kurman RJ: Serous borderline (low malignant potential, atypical proliferative) ovarian tumors: Workshop perspectives. Hum Pathol 35: 934-948, 2004

31. Longacre TA, McKenney JK, Tazelaar HD, Kempson RL and Hendrickson MR: Ovarian serous tumors of low malignant potential (borderline tumors): Outcome-based study of 276 patients with long-term ( $>$ or $=5$-year) follow-up. Am J Surg Pathol 29: 707-723, 2005

32. O'Neill CJ, McBride HA, Connolly LE, Deavers MT, Malpica A and McCluggage WG: High-grade ovarian serous carcinoma exhibits significantly higher p16 expression than low-grade serous carcinoma and serous borderline tumour. Histopathology 50: 773-779, 2007.

33. Vang R, Levine DA, Soslow RA, Zaloudek C, Shih IeM and Kurman RJ: Molecular alterations of TP53 are a defining feature of ovarian high-grade serous carcinoma: A rereview of cases lacking TP53 mutations in the Cancer Genome Atlas Ovarian Study. Int J Gynecol Pathol 35: 48-55, 2016.

34. Sundov D, Caric A, Mrklic I, Gugic D, Capkun V, Hofman ID, Mise BP and Tomic S: P53, MAPK, topoisomerase II alpha and Ki67 immunohistochemical expression and KRAS/BRAF mutation in ovarian serous carcinomas. Diagn Pathol 8: 21, 2013.

35. Singer G, Stöhr R, Cope L, Dehari R, Hartmann A, Cao DF, Wang TL, Kurman RJ and Shih IeM: Patterns of p53 mutations separate ovarian serous borderline tumors and low- and high-grade carcinomas and provide support for a new model of ovarian carcinogenesis: A mutational analysis with immunohistochemical correlation. Am J Surg Pathol 29: 218-224, 2005. 